Yusaku Yamamoto*

\title{
On eigenvalues of a matrix arising in energy-preserving/dissipative continuous-stage Runge-Kutta methods
}

https://doi.org/10.1515/spma-2021-0101

Received March 21, 2021; accepted May 29, 2021

Abstract: In this short note, we define an $s \times s$ matrix $K_{s}$ constructed from the Hilbert matrix $H_{s}=\left(\frac{1}{i+j-1}\right)_{i, j=1}^{S}$ and prove that it has at least one pair of complex eigenvalues when $s \geq 2$. $K_{s}$ is a matrix related to the AVF collocation method, which is an energy-preserving/dissipative numerical method for ordinary differential equations, and our result gives a matrix-theoretical proof that the method does not have large-grain parallelism when its order is larger than or equal to 4.

Keywords: Hilbert matrix, eigenvalue, structure-preserving numerical methods, energy-preserving, continuous Runge-Kutta methods

PACS: 02.10.Ud, 02.10.Yn, 02.30.Hq, 02.60.-X

\section{Introduction}

Let $H_{s} \in \mathbb{R}^{s \times s}$ be the Hilbert matrix of order $s$ defined by

$$
H_{S}=\left[\begin{array}{cccc}
1 & \frac{1}{2} & \cdots & \frac{1}{s} \\
\frac{1}{2} & \frac{1}{3} & \cdots & \frac{1}{s+1} \\
\vdots & \vdots & \ddots & \vdots \\
\frac{1}{s} & \frac{1}{s+1} & \cdots & \frac{1}{2 s-1}
\end{array}\right]=\left[\mathbf{h}_{1} \mathbf{h}_{2} \cdots \mathbf{h}_{s}\right]
$$

and $H_{s}^{<}$its shifted version:

$$
H_{S}^{<}=\left[\begin{array}{cccc}
\frac{1}{2} & \frac{1}{3} & \cdots & \frac{1}{s+1} \\
\frac{1}{3} & \frac{1}{4} & \cdots & \frac{1}{s+2} \\
\vdots & \vdots & \ddots & \vdots \\
\frac{1}{s+1} & \frac{1}{s+2} & \cdots & \frac{1}{2 s}
\end{array}\right]=\left[\mathbf{h}_{2} \mathbf{h}_{3} \cdots \mathbf{h}_{s+1}\right] .
$$

In this paper, we consider a matrix $K_{s}$ defined by

$$
K_{s}=\left(H_{s}^{<}\right)^{-1} H_{s}\left[\begin{array}{cccc}
1 & & & \\
& 2 & & \\
& & \ddots & \\
& & & s
\end{array}\right]
$$

and study whether it has complex eigenvalues or not.

This problem is related to the analysis of so-called structure-preserving numerical methods for ordinary differential equations (ODEs) [1-3]. More specifically, the matrix $K_{s}$ arises in the analysis of the AVF

\footnotetext{
^Corresponding Author: Yusaku Yamamoto: The University of Electro-Communications, Tokyo, Japan
} 
collocation method [4], which is a class of energy-preserving/dissipative numerical methods belonging to continuous-stage Runge-Kutta methods. As will be detailed in the next section, if $K_{S}$ has only real eigenvalues and is diagonalizable for some $s$, then the AVF collocation method of order $2 s$ has large-grain parallelism, which is a desirable property from the viewpoint of high performance computing. Actually, it is possible to analyze this problem in a different way, by using the relationship between the AVF collocation method and Gauss-Legendre Runge-Kutta method and exploiting the properties of the latter [5, 6]. But a more direct analysis based on matrix theory will be desirable because such an analysis will be applicable also to some generalizations of the AVF collocation method [7]. We therefore deal with this problem in the present paper.

The rest of this paper is structured as follows. In Section 2, we introduce the AVF collocation method and explain how its characteristics are governed by the Hilbert matrix $H_{s}$ and the matrix $K_{s}$. In Section 3, we analyze the eigenvalues of $K_{s}$ and show that it has at least one pair of complex eigenvalues for $s \geq 2$. Section 4 gives some discussion and conclusion.

\section{The matrices $H_{s}$ and $K_{s}$ and the properties of the AVF collocation method}

Let us consider the following system of ODEs:

$$
\frac{\mathrm{d} \mathbf{y}}{\mathrm{d} t}=\mathbf{f}(\mathbf{y}), \quad \mathbf{y}\left(t_{0}\right)=\mathbf{y}_{0} \in \mathbb{R}^{N},
$$

where

$$
\mathbf{f}(\mathbf{y})=S \nabla H(\mathbf{y}), \quad S \in \mathbb{R}^{N \times N}, \quad H: \mathbb{R}^{N} \rightarrow \mathbb{R} .
$$

It is well known that the system (4) is energy-conserving $(\mathrm{d} H / \mathrm{d} t=0)$ when $S$ is skew-symmetric and dissipative $(\mathrm{d} H / \mathrm{d} t \leq 0)$ when $S$ is symmetric negative semidefinite [2]. If the system (4) is discretized by general-purpose numerical integrators such as Runge-Kutta methods, however, such properties are usually lost. To resolve this problem, structure-preserving numerical integrators that preserve the energyconserving/dissipative property even after discretization have been actively studied [1-3]. One of the schemes to derive such integrators in a unified manner is continuous-stage Runge-Kutta (CSRK) methods [4, 7, 8]. In this method, one computes the numerical solution $\mathbf{y}_{1}$ at time $t+h$ from $\mathbf{y}_{0}$ at time $t$ by the following formula:

$$
\begin{aligned}
& \mathbf{Y}_{\tau}=\mathbf{y}_{0}+h \int_{0}^{1} A_{\tau, \zeta} \mathbf{f}\left(\mathbf{Y}_{\zeta}\right) \mathrm{d} \zeta, \\
& \mathbf{y}_{1}=\mathbf{y}_{0}+h \int_{0}^{1} B_{\tau} \mathbf{f}\left(\mathbf{Y}_{\tau}\right) \mathrm{d} \tau .
\end{aligned}
$$

Here, $A_{\tau, \zeta}$ is a polynomial of order $s$ in $\tau$ and order $s-1$ in $\zeta$ defined by

$$
A_{\tau, \zeta}=\left[\begin{array}{llll}
\tau & \frac{\tau^{2}}{2} & \cdots & \frac{\tau^{s}}{s}
\end{array}\right] M\left[\begin{array}{c}
1 \\
\zeta \\
\vdots \\
\zeta^{s-1}
\end{array}\right],
$$

where $M$ is an $s \times s$ matrix that defines a specific CSRK method, and $B_{\zeta}=A_{1, \zeta}$. By choosing $A_{\tau, \zeta}$ in this way and approximating the integral on the right-hand side of (6) by numerical quadrature, the integral equation (6) is reduced to a nonlinear equation, which can be solved by, for example, the simplified Newton method. The characteristics of a specific CSRK method is determined by the matrix $M$ as follows (see [7] for details).

1. If $M$ is symmetric, the corresponding CSRK method is energy-preserving. If, in addition, $M$ is positive semidefinite, the method is also dissipative when applied to dissipative ODEs. 
2. If $M$ satisfies the following equations, the corresponding CSRK method has order $2 \eta$, i.e., its local error is $O\left(h^{2 \eta+1}\right)$ :

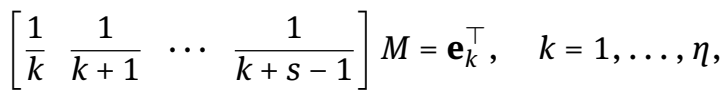

where $\mathbf{e}_{k}$ is the $k$ th column of the identity matrix of order $s$. Note that this is a sufficient condition.

3. If the eigenvalues of the matrix $E_{s} \in \mathbb{R}^{s \times s}$ defined by

$$
E_{S}=\left[\begin{array}{cccc}
1 & & & \\
& \frac{1}{2} & & \\
& & \ddots & \\
& & & \frac{1}{s}
\end{array}\right] M H_{s}^{<}
$$

are all real and $E_{S}$ is diagonalizable, then the corresponding CSRK method has large-grain parallelism. This means that the linear simultaneous equations of order $s N$ to be solved at each time step is decomposed into $s$ independent linear simultaneous equations of order $N$ each.

If we set $M=H_{s}^{-1}$, the condition (9) is satisfied for $k=1,2, \ldots, s$ and the resulting CSRK method has order $2 s$. This is called the AVF collocation method [4]. This method is energy-preserving since $H_{s}$ (and therefore $H_{S}^{-1}$ ) is symmetric and also dissipative since $H_{S}$ (and therefore $H_{S}^{-1}$ ) is positive definite. So, it is of interest to know whether the matrix $E_{s}=\operatorname{diag}\left(1, \frac{1}{2}, \ldots, \frac{1}{s}\right) H_{S}^{-1} H_{S}^{<}$has all real eigenvalues, since then the method also has the desirable property of large-grain parallelism.

Actually, it is known that the matrix $E_{s}$ corresponding to the AVF collocation method has complex eigenvalues for $s \geq 2$, because it is similar to the $E_{s}$ matrix of Gauss-Legendre Runge-Kutta method and the latter is known to have at most one real eigenvalue [6]. However, this is an indirect proof and a more direct proof based on matrix theory is desirable, since such a proof could be extended to modifications of the AVF collocation method, for which the matrix $M$ is a slight perturbation of $H_{s}^{-1}$. In the next section, we study the existence of complex eigenvalues of $K_{S}=E_{s}^{-1}=\left(H_{S}^{<}\right)^{-1} H_{s} \operatorname{diag}(1,2, \ldots, s)$ based purely on matrix theory.

\section{The eigenvalues of $K_{s}$}

\subsection{The entries of $\left(H_{s}^{<}\right)^{-1} H_{s}$}

To study the eigenvalues of $K_{s}$, we first calculate the entries of $\left(H_{s}^{<}\right)^{-1} H_{s}$ using the properties of the Hilbert matrix. Since the $2 \mathrm{nd}, 3 \mathrm{rd}, \ldots$, sth columns of $H_{s}$ is the 1 st, 2 nd, ..., $(s-1)$ th columns of $H_{s}^{<}$, respectively, it is clear that the $i$ th column of $\left(H_{s}^{<}\right)^{-1} H_{s}$ is $\mathbf{e}_{i-1}$ for $2 \leq i \leq s$. Now, let the 1st column of $\left(H_{s}^{<}\right)^{-1} H_{s}$ be $\mathbf{b}=$ $\left[b_{1}, b_{2}, \ldots, b_{s}\right]^{\top}$. Then, since $\mathbf{b}$ is the solution of the linear simultaneous equation

$$
H_{S}^{<} \mathbf{b}=\mathbf{h}_{1} \text {, }
$$

we have from Cramer's formula,

$$
\begin{aligned}
b_{i} & =\frac{\left|\mathbf{h}_{2} \mathbf{h}_{3} \cdots \mathbf{h}_{i} \mathbf{h}_{1} \mathbf{h}_{i+2} \mathbf{h}_{i+3} \cdots, \mathbf{h}_{s+1}\right|}{\left|H_{s}^{<}\right|} \\
& =(-1)^{i-1} \frac{\left|\mathbf{h}_{1} \mathbf{h}_{2} \mathbf{h}_{3} \cdots \mathbf{h}_{i} \mathbf{h}_{i+2} \mathbf{h}_{i+3} \cdots, \mathbf{h}_{s+1}\right|}{\left|H_{S}^{<}\right|} \quad(i=1,2, \ldots, s) .
\end{aligned}
$$

Note that the numerator of the last expression is the determinant of a matrix obtained by deleting the $(s+1)$ th row and the $(i+1)$ th column of $H_{s+1}$, while the denominator is the determinant of a matrix obtained by deleting the $(s+1)$ th row and the 1st column of $H_{s+1}$. Hence, the numerator and the denominator are the $(s+1, i+1)$ and $(s+1, i)$ cofactors of $H_{s+1}$, respectively, and can be written as

$$
\left|\mathbf{h}_{1} \mathbf{h}_{2} \mathbf{h}_{3} \cdots \mathbf{h}_{i} \mathbf{h}_{i+2} \mathbf{h}_{i+3} \cdots, \mathbf{h}_{s+1}\right|=\left(H_{s+1}^{-1}\right)_{i+1, s+1} \cdot\left|H_{s+1}\right| \cdot(-1)^{(i+1)+(s+1)},
$$




$$
\left|H_{S}^{<}\right|=\left(H_{s+1}^{-1}\right)_{1, s+1} \cdot\left|H_{s+1}\right| \cdot(-1)^{1+(s+1)} .
$$

By inserting these expressions into (12) and using the formula for the inverse of the Hilbert matrix [9]:

$$
\left(H_{N}^{-1}\right)_{m, n}=\frac{(-1)^{m+n}}{m+n-1} \cdot \frac{(n+N-1) !(m+N-1) !}{((n-1) !)^{2}(N-n) !((m-1) !)^{2}(N-m) !},
$$

we have

$$
\begin{aligned}
b_{i} & =-\frac{\left(H_{s+1}^{-1}\right)_{i+1, s+1}}{\left(H_{s+1}^{-1}\right)_{1, s+1}} \\
& =-\frac{\frac{(-1)^{(i+1)+(s+1)}}{i+s+1} \cdot \frac{(2 s+1) !(s+i+1) !}{(s !)^{2}(i !)^{2}(s-i) !}}{\frac{(-1)^{1+(s+1)}}{s+1} \cdot \frac{(2 s+1) !(s+1) !}{(s !)^{2} s !}} \\
& =(-1)^{i+1} \frac{(s+i) !}{(i !)^{2}(s-i) !} \quad(i=1,2, \ldots, s) .
\end{aligned}
$$

Thus, we have succeeded in computing all the entries of $\left(H_{s}^{<}\right)^{-1} H_{s}$.

\subsection{Existence of complex eigenvalues}

Now, let us apply a similarity transformation $C_{S}=D K_{S} D^{-1}$ to $K_{S}$ using a diagonal matrix $D=$ $\operatorname{diag}(1 !, 2 !, \ldots, s !)$. Then we obtain

$$
C_{s}=\left[\begin{array}{ccccc}
c_{1} & 1 & & & \\
c_{2} & & 1 & & \\
\vdots & & & \ddots & \\
c_{s-1} & & & & 1 \\
c_{s} & & & &
\end{array}\right]
$$

where

$$
c_{i}=i ! b_{i}=(-1)^{i+1} \frac{(s+i) !}{i !(s-i) !} \quad(i=1,2, \ldots, s) .
$$

Since $C_{s}$ is a companion matrix, its characteristic polynomial is given as

$$
P_{S}(x)=\left|x I_{S}-C_{S}\right|=\sum_{i=0}^{s} c_{S-i} x^{i} .
$$

Note that while the expression (18) for $c_{i}$ was derived for $i=1,2, \ldots, s$, it is valid also for $c_{0}$, because substituting $i=0$ into (18) gives $c_{0}=1$, which is the correct coefficient of $x^{s}$ in the characteristic polynomial.

To study the reality of the roots of $P_{s}(x)=0$, we use the following lemma due to Newton [10].

Lemma 3.1 (Newton). Assume that $n \geq 2$ and let $P(x)=\sum_{i=0}^{n}{ }_{n} C_{i} a_{i} x^{i}$. Then, for all the roots of the $n$th order algebraic equation $P(x)=0$ to be real, the following inequalities must hold:

$$
a_{i}^{2} \geq a_{i-1} a_{i+1} \quad(1 \leq i<n) .
$$

In our case, the coefficients $a_{i}$ in the lemma are given as

$$
a_{i}=\frac{c_{s-i}}{{ }_{s} C_{i}}=(-1)^{s-i+1} \frac{(2 s-i) !}{i !(s-i) !} \cdot \frac{i !(s-i) !}{s !} \cdot=(-1)^{s-i+1} \frac{(2 s-i) !}{s !} \quad(i=1,2, \ldots, s) .
$$

Hence,

$$
\frac{a_{i}^{2}}{a_{i-1} a_{i+1}}=\frac{((2 s-i) !)^{2}}{(s !)^{2}} \cdot \frac{s !}{(2 s-i+1) !} \cdot \frac{s !}{(2 s-i-1)}
$$




$$
\begin{aligned}
& =\frac{((2 s-i))^{2}}{(2 s-i+1) !(2 s-i-1) !} \\
& =\frac{((2 s-i))^{2}}{(2 s-i+1)(2 s-i) ! \cdot \frac{(2 s-i) !}{2 s-i}} \\
& =\frac{2 s-i}{2 s-i+1}<1 \quad(1 \leq i<s),
\end{aligned}
$$

which shows that the inequality (20) does not hold for any $i$.

Thus, we arrive at the following theorem.

Theorem 3.2. The matrix $K_{s}=\left(H_{s}^{<}\right)^{-1} \operatorname{diag}(1,2, \ldots, s)$ has at least one pair of complex eigenvalues for $s \geq 2$.

This translates into the following corollary on the AVF collocation method.

Corollary 3.3. The AVF collocation method does not have large-grain parallelism when $s \geq 2$.

\section{Discussion}

To prove the existence of a complex root of $P_{s}(x)=0$ using Newton's lemma, it is sufficient to show that (20) fails to hold for at least one of $1 \leq i<s$. In our case, however, (20) fails to hold for all of $1 \leq i<s$. Thus, it would not be so easy to modify $K_{s}$ so that (20) holds for all $1 \leq i<s$ by introducing only small number of parameters into $M$. On the other hand, the ratio of $a_{i}^{2} /\left(a_{i-1} a_{i+1}\right)=(2 s-i) /(2 s-i+1)$ is very close to 1 . This suggests that $K_{s}$ is very close to a matrix with all real eigenvalues ${ }^{1}$. Accordingly, it is still an open question whether we can modify $H_{S}$ so that $K_{S}$ has all real eigenvalues while retaining the energy-preservation/dissipative properties and the order conditions. Our analysis given in this paper should be a useful guideline in pursuing the research in this direction.

Acknowledgements: I thank the anonymous reviewers for valuable comments. I am also grateful to Prof. Yuto Miyatake of Osaka University, Japan, for bringing the paper [6] to my attention. This work is supported by JSPS KAKENHI Grant Numbers 16KT0016, 17H02828, 17K19966 and 19KK0255.

Data Availability Statement: Data sharing is not applicable to this article as no datasets were generated or analysed during the current study.

\section{References}

[1] E. Hairer, C. Lubich and G. Wanner: Geometric Numerical Integration: Structure-Preserving Algorithms for Ordinary Differential Equations, 2nd Ed., Springer, Berlin, 2006.

[2] D. Furihata and T. Matsuo: Discrete Variational Derivative Method: A Structure-Preserving Numerical Method for Partial Differential Equations, Chapman and Hall/CRC, 2010.

[3] S. Blanes and F. Casas: A Concise Introduction to Geometric Numerical Integration, Chapman and Hall/CRC, 2016.

[4] E. Hairer, Energy-preserving variant of collocation methods, Journal of Numerical Analysis, Industrial and Applied Mathematics, Vol. 5, pp. 73-84 (2010).

[5] Y. Miyatake, private communication.

[6] J. C. Butcher: On the implementation of implicit Runge-Kutta methods, BIT Numerical Mathematics, Vol. 16, pp. 237-240 (1976).

$\overline{1 \text { When }} a_{i}^{2} /\left(a_{i-1} a_{i+1}\right)=1$ holds for $1 \leq i<N, P(x)=0$ has a real root of multiplicity $N$. 
[7] Y. Miyatake and J. C. Butcher: A characterization of energy-preserving methods and the construction of parallel integrators for Hamiltonian systems, SIAM Journal on Numerical Analysis, Vol. 54, № 3, pp. 1993-2013 (2016).

[8] J. C. Butcher: An algebraic theory of integration methods, Mathematics of Computation, Vol. 26, pp. 79-106 (1972).

[9] Why does the inverse of the Hilbert matrix have integer entries?

https://math.stackexchange.com/questions/430060/why-does-the-inverse-of-the-hilbert-matrix-have-integer-entries

[10] I. Newton: Arithmetica universalis: Sive de compositione et resolutione arithmetica liber, Tooke, 1707. 